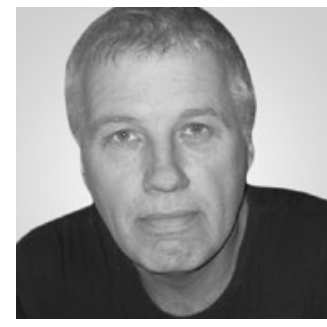

Detlef Ullenboom $* 1962$

Studium Publizistik, Sozialwissenschaft und Neuere Geschichte (M.A.), seit Mitte 2006 Redakteur von Sozial Extra. redaktion@sozialextra.de

\section{Liebe Leserin, lieber Leser,}

die meisten Erfolgsstories haben ein Kapitel, in dem eindrucksvoll beschrieben wird, wie es gelungen ist, „Betroffene zu Beteiligten" gemacht zu haben. Beteiligungs- bzw. Partzipationsmöglichkeiten scheinen großen Einfluss darauf zu haben, inwiefern man Menschen dafür gewinnen kann, sich intensiv mit einer Aufgabe zu beschäftigen - unbhängig davon, ob es dabei um die kurzfristige Mitarbeit in einem Projekt oder - grundsätzlicher - um das Verhältnis zwischen Bürger_in und demokratischem Staat geht.

In „Praxis Aktuell“ dieser Ausgabe blicken wir auf Beteiligung und Selbstorganisation in Feldern der Sozialen Arbeit, vor allem in der Kinder- und Jugendhilfe und bei Menschen mit Behinderung. Insbesondere letztere zeigen oft eine konsequent-selbstbewusste „Nichts-über-uns-ohne-uns“-Haltung, die sich über einen langen Zeitraum hinweg entwickelt hat. Beteiligung und Selbstorganisation sind allerdings keine Selbstläufer, sondern müssen zunächst gefördert und initiert werden. Um solche Prozesse in Gang zu bringen, braucht es Anknüpfungspunkte und Unterstützung im Sinne von Empowerment. Das langfristige Ziel ist, dass Menschen ihr Leben selbstständig führen und ihre Interessen selbst vertreten können. Dabei muss dann auch immer die Frage der Selbstverantwortung thematisiert werden.

Auch in unserem zweiten Schwerpunkt stehen Kinder und Jugendliche im Mittelpunkt: in letzter Zeit werden seit langem gebräuchliche, akzeptierte Standards in der Kinder- und Jugendhilfe immer wieder hinterfragt, meist begründet mit dem Hinweis auf nicht (mehr) ausreichend vorhandenen Finanzmittel. Die jeweiligen Kostenträger möchten vermeintlich teuere Maßnahmen auf den Prüfstand stellen, um ihnen nicht ausreichende Wirksamkeit nachzuweisen. Oft zeigt sich bei genauerem Hinsehen aber, dass die meisten Standards sinnvoll und notwendig sind. Nichtsdestotrotz ist die fachliche Diskussion um eine gezielte Verwendung von Mitteln nicht sakrosant, sondern für eine Weiterentwicklung unverzichtbar.

Ihnen eine erkenntnisreiche Lektüre und angenehme Ostertage Detlef Ullenboom 